\title{
Charcot und die Ätiologie der Neurosen*
}

Aus dem Medizinhistorischen Institut der Universität Zürich

(Direktor: Prof. Dr. E.H.ACKerknecht)

Von Esther Fischer-Homberger

Die Frage nach der Ursache der Neurosen war, als Jean Martin Charcot (1825-93) sich ihr in den siebziger und achtziger Jahren des 19. Jahrhunderts widmete, hochaktuell. Dies nicht nur, weil man darauf keine Antwort hatte - Unkenntnis der Dinge allein muß bekanntlich keine Neugierde wecken -, sondern auch, weil gerade um jene Zeit die Ätiologie der Krankheiten überhaupt brennend interessierte.

Führen wir uns die Situation kurz vor Augen. Die «Neurose» ist ein medizinischer Begriff. Er stammt von William Cullen aus den siebziger Jahren des 18. Jahrhunderts. Neurosen waren für Cullen alle Krankheiten, die ihre unmittelbare Ursache in Störungen der Nervenfunktion hatten. Entsprechend der prädominanten Bedeutung, die man im 18. Jahrhundert der Neurophysiologie zumaß, fand Cullen, daß man eigentlich alle Krankheiten zu den Neurosen zählen könnte. Er selber gebrauchte den Namen «Neuroses» indessen nur für diejenigen Krankheiten, «welche auf das Nervensystem fast nur allein, oder doch zuerst und vornehmlich wirken ...» ${ }^{1}$ Und das waren alle möglichen Krankheiten, die man bis dahin zum Formenkreis der Hysterie und der Hypochondrie gerechnet hatte. Die Störungen, die Cullens Neurosen zugrunde lagen, waren physiologischer Natur.

Im Spannungsfeld der im 19. Jahrhundert mächtig auf kommenden pathologischen Anatomie spalteten die Neurosen Cullens sich dann - sofern sie überhaupt noch auf nervliche Störungen zurückgeführt wurden - in zwei Gruppen auf: Bei der einen Gruppe fand man, pathologisch-anatomisch, Läsionen des Nervensystems, bei der anderen keine Befunde, die das jeweilige Krankheitsbild erklärt hätten. Dabei ergab es sich - PINEL machte den Anfang - daß der Name «Neurosen » an der letzteren Gruppe hängenblieb; für die pathologisch-anatomisch aufgeklärten Krankheiten fanden sich wie von selbst spezifischere Namen. Man blieb nun zwar dabei, anzunehmen,

* Frau Prof. Dr. Erna Lesky zum 60. Geburtstag am 22. Mai 1971 gewidmet.

1 William Cullen, Kurzer Inbegriff der medicinischen Nosologie, nach der 3. Ausgabe, Leipzig 1786, Band 1, S. 249 ff. Vgl. auch Esther Fischer-Homberger, Hypochondrie, Bern 1970, S. 78-79. 
daß den Neurosen etwas Körperliches zugrundeliege. Man kam aber mehr und mehr überein, daß dieses Substrat eben noch unbekannt oder überhaupt unerkennbar sei. Man nahm nun noch unentdeckte oder vielleicht submikroskopische Läsionen, molekulare Störungen, Vergiftungen, Degeneration des Nervensystems, unerkannte Veränderungen des nervösen Apparates, die dessen Funktion behinderten, als Ursachen der Neurosen an, aber man war sich im allgemeinen bewußt, daß es sich dabei um Spekulationen handelte. VIRCHOW sagte $(1855 / 56)$ in seinen Vorlesungen über allgemeine pathologische Anatomie über Krankheiten, die pathologisch-anatomisch nicht aufgeklärt werden könnten: «Hier kann man dann allerdings einen gewissen Spielraum lassen für die Spekulation. Und auch wir machen hier eine Spekulation, indem wir schließen, daß auch in diesen Fällen eine molekulare Störung existiere ... ${ }^{2}$ «Das centrale Nervensystem kann vielleicht dephosphorisiert werden ..., vielleicht geht es auch leichte, nicht nachweisbare krankhafte Veränderungen in seiner chemischen Struktur ein ...» schreiben Beard und Rockwell 1871 über die Pathologie der von ihnen eingeführten Neurasthenie ${ }^{3}$. Die Hysterie, die andere große Neurose des letzten Jahrhunderts, war eine dem Blick der pathologischen Anatomie womöglich noch undurchsichtigere Affektion, wie dies schon BRIQUET 1859 in seiner klassischen Abhandlung über die Hysterie hervorgehoben hat ${ }^{4}$. Nachdem die Zurückführung der Hysterie auf genitale Läsionen, die noch GriesINGER geläufig gewesen ist ${ }^{5}$, in Mißkredit gefallen war, herrschte im Gebiet der Hysterieforschung eine besondere Ratlosigkeit bezüglich der medizinisch-naturwissenschaftlichen Grundlagen dieses Leidens. Man sprach ganz allgemein von der Dunkelheit, die das Wesen der Hysterie verhülle.

Es ist dem Leser sicher aufgefallen, da $\beta$ wir bis dahin Krankheitsursache und körperliches Substrat stillschweigend eng assoziiert haben. Diese Asso-

2 Rudolf Virchow, Die Vorlesungen über Allgemeine Pathologische Anatomie aus dem Wintersemester 1855/56 in Würzburg, Jena 1930, S. 35.

3 George M.Beard und A.Rockwell, Practische Abhandlung über die medicinische und chirurgische Verwerthung der Elektrizität, deutsch bearbeitet von Dr. R. VAẹter, Prag 1874, S. 255.

4 Zitiert nach Jean-Marie Bruttin, Différentes théories sur l'hystérie dans la première moitié du XIX siècle (= Zürcher medizingeschichtliche Abhandlungen, herausgegeben von E. H. Ackerknecht, N.R. Nr. 66) Zürich 1969, S. 43-44.

5 Vgl. Wilhelm Griesinger, Die Pathologie und Therapie der psychischen Krankheiten für Ärzte und Studierende, 2. Auflage, Stuttgart 1867, S. 207: «Das Licht, das durch das Speculum fällt, wird, wie es die Hysterie so wohl beleuchtet, auch vieles der Hysterie nahestehendes Irresein allein zu erhellen vermögen!» 
ziation ist natürlich keine unfragwürdige. Hatte nicht auch die körperliche Veränderung, die einer Krankheit zugrunde lag, ihre Ursachen? Mußte der körperliche Befund Ursache der krankhaften Erscheinungen sein? Gab es nicht neben ihr Faktoren aus nichtmedizinischen Bereichen, die die Krankheit ursächlich bedingten? War nicht der Ursachenbegriff selbst überhaupt so komplex, daß er prinzipiell nicht an einzelne Befunde, sondern nur an Wirkungsschemata geknüpft werden konnte?

Die Problematik des Ursachenbegriffes war den Ärzten des 19. Jahrhunderts natürlich wohlbekannt. Da, wo die pathologisch-anatomische Methode aber positive Resultate zeitigte, trat diese Problematik stark in den Hintergrund. $\mathrm{Da}$, wo zu umrissenen Krankheitsbildern regelmäßig spezifische pathologisch-anatomische Befunde erhoben wurden, aus denen sich die beobachtete Symptomatik erklären ließ, neigte man dazu, diese Befunde mit der Krankheitsursache zu identifizieren. Morgagni hat sein Werk in aller Selbstverständlichkeit nicht nur «de sedibus ...» sondern auch «... et causis» morborum genannt. Eine Verstärkung der Assoziation von Krankheitsursache und pathologisch-anatomischem Substrat ergab sich auch aus der chirurgischen Erfahrung, daß vielfach mit der Entfernung der pathologischen Veränderung die Krankheit verschwand. Der Punkt, an dem eine Krankheit therapeutisch erfolgreich angegangen werden kann, pflegt schon dadurch mit der Ursache dieser Krankheit in engen Zusammenhang gestellt zu werden. Da das lokalisierende medizinische Denken des 19. Jahrhunderts überhaupt unter dem Einfluß der Chirurgie aufgekommen ist, war körperliches Substrat und Krankheitsursache sogar zum vornherein assoziiert ${ }^{6}$.

Wo nun aber das pathologisch-anatomische Denkmodell durch keine positiven Befunde gestützt wurde, geriet es leicht ins Schwanken. Die ganze Problematik des Ursachenbegriffs brach dann wieder auf. Einfache und verzweigte Ursachenketten mit unbekannten Gliedern traten dann an die Stelle des simplen Ursache-Folge-Schemas. Und weil die Neurosen im Rahmen des pathologisch-anatomischen Denkens gerade als Affektionen unbekannten Ursprungs definiert waren, war dies im Gebiet der Neurosen ganz ausgeprägt der Fall. Außer der unbekannten körperlichen Ursache traten daher alle möglichen anderen Dinge als bekannte Ursachen der Neurosen auf. Die Doktrin von der Degeneration verdankt ihre große Blüte in der

6 Vgl. Erwin H.Ackerknecht, Medicine at the Paris Hospital 1794-1848, Baltimore 1967, S. 25, und OwsEI Temkin, The role of surgery in the rise of modern medical thought, Bull. Hist. Med. 25 (1951) 248-259. 
Psychiatrie u.a. diesen Umständen. Aber auch auf nichtmedizinische Ursachen wurden die Neurosen zurückgeführt. Auch Ärzte haben ein Kausalitätsbedürfnis.

So haben wir unseren anfänglichen Ausführungen über die Unbekanntheit der Ätiologie der Neurosen beizufügen : Im Rahmen eines pathologischanatomisch orientierten Denkens war die Ursache der Neurosen unbekannt. Anderen Denkarten hingegen stellte sie sich als bekannt dar. Indem BEARD zum Beispiel die Neurasthenie soziologisch betrachtete, erschienen ihm «der Druck der Entbehrungen, Geschäfts- und Familiensorgen ... plötzliches Aufgeben des Geschäfts ...» als ihre Ursachen ${ }^{7}$. Indem Briquet die Hysterie psychologisch betrachtete, fand er in Eigentümlichkeiten des Gefühlslebens, in der Langeweile, in Kummer, Liebe, psychischem Schock Ursachen dieses Leidens ${ }^{8}$. Sowohl mit der soziologischen als auch mit der psychologischen Betrachtungsweise der Neurosen knüpften diese Autoren an uralte Tradition an - schon die Vorläufer der Neurosen sind immer wieder als Zivilisationskrankheiten und als durch Seelisches verursacht beschrieben worden ${ }^{9}$.

Man könnte sich vorstellen, daß die psychologischen und die soziologischen Ursachen der Neurosen dem Bedürfnis der Mediziner genügt hätten. Dies ist von Stahl bis zu einzelnen modernen Psychosomatikern und Sozialpsychiatern immer wieder der Fall gewesen. In der Zeit, von der wir hier sprechen, galten solche Lösungen aber letztlich nicht. Durch die Erfolge in der Technik und in der Medizin verstärkt, hatte das materialistisch-naturwissenschaftliche Denken im Laufe des 19. Jahrhunderts gegenüber anderen Denkarten eine solche Dominanz und Verbindlichkeit erreicht, daß andere als materialistisch-naturwissenschaftlich faßbare Krankheitsursachen vom durchschnittlichen Mediziner gar nicht als solche angenommen werden konnten. Neurologie und Psychiatrie, medizinische Psychologie und Körpermedizin hingen nicht nur äußerlich eng zusammen, sie bewegten sich auch im gleichen denkerischen Koordinatensystem, in dem es keine regelrechte Krankheitsursache geben konnte, die nicht materieller Natur war.

Die Ärzte allerdings - auch der Neuroseforscher СнARcot wird dazugehören - die sich speziell mit den Krankheiten ohne bekannte körperliche Ursache befaßten, fühlten sich in diesem System begreiflicherweise nicht

7 BEARD und Rockwell, S. 256.

8 Zitiert nach BrUtTin, S. 34-37.

9 Vgl. Fischer-Homberger, bes. S. 38-44. 
wohl. Und gerade zu Charcots Zeit wurde die Unkenntnis der Neuroseätiologie als besonderer Mangel empfunden. Denn nicht nur drang der schon von LeNNEC, dann 1853 von Rilliet und BARTHEZ ausgestoßene Schrei nach der Kenntnis der Krankheitsursachen ${ }^{10}$ mehr und mehr an das allgemeine Medizinerohr. Nicht nur fiel gerade in jener Zeit die Morelsche Degenerationstheorie allmählich in Mißkredit ${ }^{11}$, welche doch bis dahin noch eine mehr oder weniger anerkannte medizinische Ätiologie der Neurosen geliefert hatte. Es nahm auch gerade damals die Bakteriologie ihren unerhörten Aufschwung ${ }^{12}$. Und die Bakteriologie war in vorher nie dagewesenem Maße geeignet, ätiologisches Denken in die Medizin zu bringen. Die Forschung à la mode war nun die bakteriologische Forschung, und die bakteriologische Forschung wurde als eine Erforschung der wahren Krankheitsätiologien aufgefaßt. Hellpach schreibt 1904 rückblickend über die Bakteriologie jener Zeit als von einer «modischen Strömung, welche in ihrer Nährgelatine die Krankheitsursachen eingefangen zu haben meinte», ${ }^{13}$ MArtius sah ihren theoretischen Hauptfehler «in einer durchaus naiven Anwendung des Ursachenbegriffes ${ }^{14}$ "W $\mathrm{W} h r e n d$ wir uns bisher größtenteils auf die Feststellung des klinischen und anatomischen Tatbestands beschränkt hatten, traten nun mit einem Mal die ätiologische Denkweise und die Fragen nach der Entstehung ... aller einzelnen Krankheitserscheinungen vollständig in den Vordergrund» schreibt Struempeld, im Sinne des damaligen Selbstempfindens der Mediziner zugespitzt, über die Jahre nach $1878^{15}$.

Dies ist der Hintergrund, auf dem ich über Charcots Ansichten zur Ätiologie der Neurosen berichten möchte.

Die Neurose, die Charcot vor allem untersuchte, war bekanntlich die Hysterie. 1870 übernahm er in der Salpêtrière eine Abteilung mit einer groBen Zahl von Frauen, die an Krämpfen litten, die teils epileptischer Natur, teils hysterisch waren ${ }^{16}$.

10 Ackerknecht, Paris Hospital, S. 122.

11 Erwin H. Ackerknecht, Kurze Geschichte der Psychiatrie, 2. Auflage, Stuttgart 1967, S. 57.

12 Vgl. Erwin H. Ackerknecht, A short history of medicine, 2. Auflage, New York 1968, S. 180.

13 Willy Hellpach, Grundlinien einer Psychologie der Hysterie, Leipzig 1904, S. 69.

14 Friedrich Martius, Konstitution und Vererbung, Berlin 1914, S. 21.

15 Adolf Struempell, Aus dem Leben eines deutschen Klinikers, 2. Auflage, Leipzig 1925, S. 118.

16 Henri F. Ellenberger, The discovery of the unconscious, New York 1970, S. 90. 
Zunächst ging er an diese Krankheitsbilder ganz als Neurologe und Neuropathologe heran. Durch die Methode der klinischen und pathologisch-anatomischen Beobachtung war es ihm zwischen 1862 und 1870 gelungen, alle die Entdeckungen auf dem Gebiet der disseminierten Sklerose, Paralysis agitans und Tabes dorsalis zu machen, die ihm den Ruf des ersten Neurologen seiner Zeit eingetragen haben. Diese Methoden wendete er nun auch auf die Hysterie an. Er bemühte sich zunächst um ihre genaue Beschreibung. Er brach mit der Tradition, die die Hysterie einen Proteus nannte, der beliebige Form annehmen könne, und fand ganz bestimmte Gesetzmäßigkeiten in ihrer Symptomatik und ihrem Verlauf. Er versuchte auch mit der Tradition zu brechen, die kein pathologisch-anatomisches Substrat der Neurosen kennen wollte und fand eine Sklerose der Seitenstränge bei prolongierter hysterischer Kontraktur ${ }^{17}$ und veränderten Augenfundus bei hysterischer Amaurose ${ }^{18}$. Die Pathologie ließ ihn im folgenden aber im Stich. Die erwartete Symptomatik der Hysterie konnte Charcot dank bewußter und unbewußter Hilfe seiner Patienten und Assistenten zeitlebens beobachten, die pathologische Anatomie aber konnte sich nicht nach seinen Erwartungen richten. Снarcot mußte als pathologischer Anatom zum wenigsagenden Schluß kommen, der Hysterie liege eine Läsion «sine materia» im Nervensystem oder eine «lésion dynamique» zugrunde ${ }^{19}$. So war der sieggewohnte Forscher in der peinlichen Situation, seine Methode versagen sehen zu müssen. Er sah sich nach neuen Methoden, die Hysterie zu untersuchen, um. Als solche bot sich ihm die damals gerade vieldiskutierte Hypnose. Es scheint unbekannt, inwieweit Сharcot das Studium der Hypnose $1878 \mathrm{im}$ Hinblick auf die Probleme der Hysterie aufgenommen hat. Jedenfalls kam er bald zur Auffassung, daß die Hypnose eine Neurose sei, die nur bei Hysterikern überhaupt zu beobachten sei, und daß er in ihr einen experimentellen Zugang zur Hysterie gefunden habe. In einem gewissen Stadium der Hypnose ließen sich hysterische Symptome reproduzieren und manipulieren. Der Wirkungsmechanismus war dabei die Suggestion.

Auf dem Weg der Suggestion konnten beliebige Vorstellungen und Willensrichtungen des Hypnotiseurs am Hypnotisierten bis in die Körpersphäre hinein wirksam werden. Sollten es nun bei der Hysterie solche Vorstellun-

17 Jean-Martin Charcot, Leçons sur les maladies du sytème nerveux faites à la Salpêtrière, Paris $1872-1873$, S. 317.

18 Ebenda, planche VIII.

19 Jean-Nartin Charcot, Leçons sur les maladies du système nerveux faites à la Salpêtrière, Band 3, Paris 1887, S. 16, 288. 
gen sein, die pathogen wirkten ? Sollte Suggestion an der hysterischen Symptombildung beteiligt sein ? Der Gedanke war naheliegend. Schon historisch waren Zusammenhänge zwischen Hysterie und Hypnose gegeben. Viele hergebrachterweise zur Hysterie gehörige Phänomene hätten so plausibel durchleuchtet werden können. Charcot selbst kannte das Phänomen der hysterischen Epidemien und fand in der Isolierung ein adäquates therapeutisches Mittel gegen Hysterie ${ }^{20}$. Wäre so nicht auch die traditionelle Ansicht, die Hysterie könne jede Form annehmen, verständlich geworden ?

Aber das war gerade einer von den Punkten, die Charcot daran hindern mußten, Vorstellungen, Gefühlen, Strebungen in der ganzen Hysterielehre ursächliche Bedeutung zuzumessen, die Suggestion zum allgemeinen Mechanismus in der Hysterieentstehung zu machen. Снarcot hatte ja die Proteushaftigkeit der Hysterie eben gerade nicht gefunden, er kannte ja eben gerade ihre ganz bestimmte, von Ort, Zeit und Umständen unabhängige Symptomatik, ihren Verlauf. Er hatte zwar die Läsionen, die ihr zugrunde lagen, nie gesehen, aber er wußte sie zu lokalisieren wie die der multiplen Sklerose oder anderer neurologischer Krankheiten. Die Annahme pathogener Vorstellungen hätte in diesem System nur störend gewirkt.

Zweitens wäre es im Rahmen von Снавсотs Konzept nötig gewesen, daß bei Hysterie irgendwelche spontane hypnotische Zustände aufgetreten wären, damit die Suggestion bei ihr hätte wirksam werden können. Freud und Breuer haben später tatsächlich das Auftreten von «hypnoide[n] Zustände[n]» als ein «Grundphänomen» der hysterischen Neurose angenommen ${ }^{21}$. Снавсот entschloß sich zu einem solchen Umbau seiner Hysterielehre nicht.

Ein Drittes mag Charcot neben Prestige- und Verbissenheitsgründen noch davon abgehalten haben, die Lehren der Hypnose zur Säule seiner Hysterielehre zu machen: er war nicht Psychologe und wollte keiner sein. Nun betrachtete er zwar die Hypnose als eine Neurose, Nervenkrankheit sine materia, und deutete die suggestiven Worte, Bewegungen, Empfindungen nicht als psychologische, sondern als physische Agentien und die dadurch hervorgerufenen Vorstellungen und Gefühle physiologisch. Trotzdem waren die Größen, mit denen er da umging, psychologische, nicht nur,

20 Ebenda, S. 235 ff., und: Jean-Martin Charcot, Poliklinische Vorträge, Band 1, Schuljahr 1887/88, Übersetzt von Sigmund Freud, Leipzig/Wien 1892, S. 137.

21 Sigmund Freud, Sammlung kleiner Schriften zur Neurosenlehre aus den Jahren 1893-1906, 2. Auflage, Leipzig/Wien 1911, S. 22-24. 
weil sie seit Locke und Herbart von der Psychologie gepachtet worden waren, sondern auch, weil sie eigentlich einem psychologischen Bezugssystem entstammten. Die psychologische Antwort auf die Frage nach der Ursache der Hysterie konnte aber für СнАвсот, der die materiellen Grundlagen der Hysterie suchte, nicht genügen. Psychologie konnte ihm höchstens Methode, aber nicht Ziel der Erkenntnis sein.

In einem Teilgebiet aber hat Снarcot psychologisches Denken doch als tragenden Teil in die Hysterielehre eingebaut: im Gebiet der traumatisch entstandenen Neurosen. Die infolge eines Unfalles entstandenen Neurosen hatten in England seit den sechziger Jahren, in Deutschland seit den siebziger Jahren, im Zusammenhang mit dem Aufkommen von Unfall-Haftpflichts- und Versicherungsgesetzen, zunehmend das Interesse von Ärzten und Nichtärzten auf sich gezogen. Einer Anregung amerikanischer Autoren (Walton, Putnam) folgend ${ }^{22}$, faßte Charcot diese Krankheitsbilder als traumatische Hysterien - das waren im wesentlichen Charcots männliche Hysterien - auf. Er befaßte sich mit ihrem Studium vor allem in den Jahren 1884 und 1885, aber auch später. Nicht zufällig hat FreUd diese Forschungen 1893 als Charcots weittragendste bezeichnet ${ }^{23}$. Auch Ellenberger rechnet sie zu Charcots «most spectacular achievements ». ${ }^{24}$ Denn in der Entstehung der traumatischen Hysterie spielten nun tatsächlich die Suggestion und die (unbewußten) pathogenen Vorstellungen führende Rollen. Und damit wurde Charcots Lehre von der traumatischen Hysterie zu einem Kristallisationskern der späteren gesamten Neurosenlehre. Charcots Modellfall der traumatischen Hysterie war die traumatisch entstandene hysterische Lähmung. Diese entstand so: Ein Patient erhielt zum Beispiel einen Schlag gegen seinen Arm. Dieses Trauma vermochte den Arm nicht ernsthaft zu verletzen, doch versetzte es den Betroffenen in einen Schreckoder «Schockzustand». Dieser Zustand, der als Analog zur Hypnose gelten kann, war dadurch charakterisiert, daß das «Ich» des Patienten benebelt und geschwächt war. In diesem Zustand war das «Ich» des Patienten außerstande, die in der Vorstellung desselben vielleicht flüchtig entstandene Idee, der vom Trauma betroffene Arm könnte nun gelähmt sein, als unrealistisch von sich zu weisen. Auf dem Weg der Autosuggestion konnte diese Idee vielmehr direkt in das unbewußte Vorstellungsleben, in das Gehirn des

22 Charcot, Leçons, Band 3, 1887, S. 250.

${ }^{23}$ Freud, Sammlung kleiner Schriften, S. 11.

${ }^{24}$ Ellenderger, S. 90. 
Patienten eindringen und sich dort festsetzen. Vom Ich unkontrolliert, konnte sie dort unbehindert wachsen wie ein Parasit, bis sie eine Größe und Stärke erreicht hatte, kraft derer sie sich tatsächlich in einer motorischen Lähmung des traumatisierten Armes realisierte ${ }^{25}$.

Eine Erklärung traumatisch-neurotischer Symptome als Folge einer irrtümlichen Idee hatte R. Reynolds schon 1869 gegeben, Снаrcot bezog sich unter anderem auch auf diese Arbeit «on paralysis, and other disorders ... dependent on idea ${ }^{26}{ }^{26}$ Was er aber neu dazubrachte, war, daß die pathogene Idee von anderen Vorstellungen abgeschnitten, unbewußt sein mußte. Сharcot steht mit dieser Lehre von der Entstehung der traumatischen Neurose sehr nahe bei der Psychogenie der Hysterie. Das zeigt auch seine Auffassung, seine Lehre werde dadurch bestätigt, daß die traumatische Lähmung sich durch ärztliche Suggestion reproduzieren und beheben lasse ${ }^{27}$. Da ist wieder Schluß von der Therapie auf die Ätiologie impliziert: in diesem Falle der Schluß von der Wirksamkeit der Psychotherapie auf die Psychogenie. Сharcot selbst faßt seine Lehre eher als psychologische denn als medizinisch-materialistische auf. «Dies ist die physiologische, oder, wenn Sie es lieber hören, psychologische Theorie, die ich für das Verständnis der localen hysterotraumatischen Lähmungen aufgestellt habe», sagt er dazu. «Ich muß gestehen, ich halte etwas auf sie, weil ich glaube, daß sie der Wahrheit ziemlich nahe kommt.» Und sein Übersetzer Freud bemerkt in einer Fußnote: «Dies ist die berühmte Theorie von der Entstehung hysterischer Lähmungen durch Autosuggestion, die Сharcot zuerst ... 1886 ausgesprochen hat.» ${ }^{28}$ Übrigens hat Снarcot diese Theorie nicht auf die traumatischen Neurosen beschränkt, er hat außer dem Trauma auch heftigen Emotionen und Giften, wie Alkohol und Haschisch, die Wirkung einer Hypnose zuerkannt ${ }^{29}$. Auch hat er in späten Jahren mit «La foi qui guérit» die Möglichkeit anerkannt, daß Suggestion in viel weiterem Maße wirksam sein könnte, als er bis dahin zugegeben hatte ${ }^{30}$.

25 Charcot, Leçons, Band 3, 1887, S. 333-369, 393-394, 441-458. Vgl. auch: J.M. Charcot, Neue Vorlesungen über die Krankheiten des Nervensystems insbesondere über Hysterie, Übersetzt von Sigmund Freud, Leipzig/Wien, 1886, S. 272-306.

26 Charcot, Leçons, Band 3, 1887, S. 335. Vgl. Russell Reynolds, Remarks on paralysis, and other disorders of motion and sensation, dependent on idea, Brit. Med. Journ. $1869 /$ II, 483-485.

27 Charcot, Leçons, Band 3, 1887, S. $336 \mathrm{ff}$.

28 Charcot, Poliklinische Vorträge, Band 1, S. 100.

${ }^{29}$ Charcot, Leçons, Band 3, 1887, S. 451.

30 Jean-Martin Charcot, La foi qui guérit, Paris 1897. 
Charcot steht mit dieser Theorie am Anfang einer nach ihm und durch ihn in Gang gekommenen "Re-Psychologisierung » der Medizin ${ }^{31}$, der Anerkennung psychologischer Methoden und Ergebnisse in der Medizin, für die der Neurologe J.P.Moebius, der ursprünglich Theologie und Philosophie studiert hatte, mit folgenden Worten eingetreten ist: «Will man aus der Unklarheit herauskommen, die gewöhnlich allen medicinischen Erörterungen über Psychisches zu Grunde liegt, so muß man ... auch den Muth haben, über die Grenzlinien, die die , exacte Wissenschaft ${ }^{6}$ gezogen hat und polizeilich bewachen läßt, hinauszugehen... In der That wird die bisherige Beschränkung der Medicin nicht bleiben. Schon ist die Erkenntniß allgemein geworden, daß der Arzt ohne Psychologie nicht auskomme. ${ }^{32}$ Moebius hat denn auch 1888 den berühmt gewordenen Satz geprägt: «Hysterisch sind alle diejenigen krankhaften Veränderungen des Körpers, welche durch Vorstellungen verursacht sind.» ${ }^{33}$

Charcot hat Moebius' «Muth» aber noch nicht gehabt. Er hat sich mit der psychologischen Antwort auf eine Frage, die er als materialistischer Naturwissenschafter gestellt hatte, nicht begnügt. Er zog die spekulative Psychologie als Forschungsmethode herbei, weil sie ihm mehr zu ergeben schien als die spekulative Naturwissenschaft, aber er wollte doch letzten Endes zu einer exakt-wissenschaftlichen Lösung kommen. Er sprach von seiner psychologischen Theorie ja doch als von einer physiologischen. Sein Vergleich der pathogenen unbewußten Vorstellung mit einem Parasiten ist mehr als ein Bild: Indem das Bild an die Bakteriologie erinnert, gibt es dem psychologischen Begriff einen naturwissenschaftlichen Anstrich. Снавсот lokalisiert den Parasiten ja auch: im Cortex; im motorischen Cortex im Falle der Lähmung. Er betrachtet die Bewegungsvorstellung mit SPENCER, BAIN und Riвот als eine Vorstufe der Bewegung selbst, also als ein physiologisches Geschehen - in gewissem. Sinne ist die Vorstellung für ihn die gesuchte dynamische Läsion, die der Hysterie zugrunde liegt ${ }^{34}$. Auch die Suggestion sucht Снавсот in neurophysiologischen Terminen zu verstehen ${ }^{35}$, die Hypnose ihrerseits als eine Neurose. Die Hypnose ist denn auch nicht durch irgendwelche psychische Agentien ersetzbar, sondern durch körperliche

31 Ellenberger, S. 548.

32 Paul Julius Moebius Neurologische Beiträge, 2 Hefte, Leipzig 1894, Heft 1, S. 182.

33 Paul Julius Moebius, Über den Begriff der Hysterie, Centralblatt für Nervenheilkunde, Psychiatrie (etc.) 11 (1888) 66.

34 Сharcot, Leçons, Band 3, 1887, S. $463 \mathrm{ff}$.

35 Ebenda, S. $450 \mathrm{ff}$. 
Einwirkungen, Erschütterungen, Gifte, Schock. Und schließlich gibt es aus alledem noch keine Hysterie, wenn die Voraussetzung einer krankhaften (körperlichen) Prädisposition nicht erfüllt ist. 1891 sagt es CHarcot klar: Gifte, Emotionen, Traumen sind «agents provocateurs» der Hysterie ihre eigentliche Ursache liegt in der Prädisposition, die hereditär gegeben ist $^{36}$.

Charcot hat also eine Psychogenie der Hysterie gekannt, aber er hat nie geglaubt, damit die Ursache der Neurose entdeckt zu haben. Dazu war er auch als Psychologe zu sehr Naturwissenschafter. Es bedurfte einer Abwendung des psychologischen Denkens vom medizinisch-naturwissenschaftlichen Denken, daß diese Auffassung für falsch angesehen werden konnte. Es brauchte eine Vergegensätzlichung von Psychologie und exakter Naturwissenschaft, daß die psychologische Neuroseursache psychologischerseits einer postulierten materiellen Neuroseursache als die richtige entgegengestellt werden konnte. Eine solche Vergegensätzlichung war CHARcot und seiner Zeit weitgehend fremd. Mit Moebius, vor allem Freud, ist sie dann praktisch später aufgekommen. Aber gerade Freud hat sie theoretisch auch nicht angenommen.

Freud kritisierte Charcot zunächst (1893) nicht, weil er als wirkliche Hysterieursache nicht eine psychologische, sondern nur eine körperliche Größe annehmen wollte. Er kritisierte vor allem, daß er die Heredität als Ursache überschätzt habe, so daß kein Raum für die Erwerbung von Neuropathien übriggeblieben sei ${ }^{37}$, während er selbst dem Trauma und dem starken Affekt, später einem postulierten Gift, mehr ursächliche Funktion zuschreiben wollte. Damit stellte er Charcots Konzept nicht ein anderes, psychologisches entgegen, sondern dieses selbst, mit anderem Akzent versehen.

Später hat Freud die Neuroseätiologie dann psychologisiert, und es ist da und dort, besonders durch den Ersten Weltkrieg, die Auffassung aufgekommen, die Ursache der Neurosen habe sich in der psychologischen Nährgelatine einfangen lassen. Freud hat diese Auffassung nicht bekämpft, hat sie aber, mindestens theoretisch, nicht geteilt. Er hat nie aufgehört zu hoffen, «daß all unsere psychologischen Vorläufigkeiten einmal auf den Boden organischer Träger gestellt werden $»,{ }^{38}$ und er lehrte, da 3 «das Lehr-

36 Jean-Martin Charcot, Clinique des maladies du système nerveux, Band 1, Paris 1892, S. 291-292.

37 Freud, Sammlung kleiner Schriften, S. 13.

${ }^{38}$ Sigmund Freud, Gesammelte Werke chronologisch geordnet, Band 10, London 1940, S. 144. 
gebäude der Psychoanalyse ... irgend einmal auf sein organisches Fundament aufgesetzt werden soll». Noch 1917 hielt er der Kritik, die Psychoanalyse «bemühe sich um eine rein psychologische Theorie der neurotischen Erscheinungen, und das sei ganz aussichtslos, denn psychologische Theorien könnten nie eine Krankheit erklären», entgegen, «daß die Sexualfunktion nichts rein Seelisches ist ... ${ }^{39}$

So hat sich Charcots Frage nach einer medizinisch-naturwissenschaftlich faßbaren Neuroseätiologie selbst durch die Psychoanalyse im Grunde nicht überlebt - sie ist psychologisch nicht zu beantworten. Und was CHarcots eigene Antworten betrifft, so harren diese noch immer der Überprüfung. Die Frage nach der Ätiologie der Neurosen, wie sie Charcot gestellt hat, ist so offen, wie sie zu Cнавсотs Zeit war. Aber sie ist nicht mehr so brennend wie zu Charcots Zeit. Sie ist zum Teil durch die psychologischen Antworten verschüttet. Auch hat die Krankheitsätiologie überhaupt an Interesse etwas verloren. Man denkt heute in der Krankheitslehre weniger in Modellen von Wirkungsketten als in solchen von kompliziert verwobenen Wirkungskreisen. Entsprechend sucht man weniger mehr nach einzelnen ersten Krankheitsursachen, wie man sie zu Снавсотs Zeit in den Bakterien kurz gefunden zu haben glaubte, als nach der Vielfalt ursächlicher Faktoren und den Regeln ihres Zusammenspiels, das zur Erscheinung der Krankheit, z.B. der sogenannten Neurose, führt.

39 Freud, Gesammelte Werke, Band 11, S. 402-403. 